\title{
PROTÓTIPO DE REALIDADE AUMENTA EM DISPOSITIVOS MÓVEIS NA ABORDAGEM DE SÓLIDOS GEOMÉTRICOS
}

\section{REALITY PROTOTYPE INCREASES ON MOBILE DEVICES IN GEOMETRIC SOLIDS APPROACH}

\author{
Fernando Oliveira Garcia ${ }^{1}$, Ronaldo Celso Messias Correia ${ }^{2}$ \\ ${ }^{1}$ Universidade Estadual do Norte do Paraná - UENP. ${ }^{2}$ Universidade Estadual Paulista - \\ UNESP. \\ E-mail: fernando.uenp@hotmail.com
}

RESUMO - As Tecnologias da Informação e Comunicação inseridas no contexto do ensino de matemática podem promover uma maior participação e envolvimento dos alunos com os conteúdos abordados. Novas ferramentas tecnológicas surgem numa frequência contínua e ascendente, inseri-las no contexto educativo e aproveitar de forma otimizada seus recursos é papel da escola e seus atores. A presente pesquisa apresenta os resultados de um estudo que investiga o desenvolvimento e a utilização da Realidade Aumentada em aplicativos para dispositivos móveis nas aulas de Matemática da Educação Básica. Para tanto, desenvolveu-se um aplicativo na plataforma Android que permite a abordagem do tema: sólidos regulares, também conhecidos como sólidos de Platão. O levantamento de dados deu-se por meio de um questionário empregado junto a um grupo composto de professores de matemática e graduandos de um curso de licenciatura plena em matemática revelando que poucos tiveram contato com este tipo de aplicativo, e indica também que aliar dispositivos móveis aos recursos de realidade aumentada denota ser relevante para abordagem dos sólidos geométricos.

Palavras-chave: Ensino de Matemática; Realidade Aumentada; Aplicativos; Dispositivos móveis; Geometria.

ABSTRACT - Information and Communication Technologies inserted in the context of mathematics teaching can promote greater participation and involvement of students with the contents covered. New technological tools emerge in a continuous and rising frequency, inserting them in the educational context and making optimal use of their resources is the role of the school and its actors. This research presents the results of a study that investigates the development and use of Augmented Reality in mobile applications in Basic Education Mathematics classes. To this end, an application was developed on the Android platform that allows the approach of the theme: regular solids, also known as Plato solids. The survey was conducted through a questionnaire used by a group of math teachers and undergraduate students of a full degree course in mathematics revealing that few had contact with this type of application, and also indicates that to combine mobile devices augmented reality resources denotes being relevant for approaching geometric solids.

Keywords: Mathematics Teaching; Augmented Reality; Applications; Mobile Devices; Geometry. 


\section{INTRODUÇÃO}

O presente trabalho é um recorte de uma pesquisa de mestrado que investiga o uso de novos recursos de tecnologia para inseri-los na prática docente, impulsionado pelos diversos trabalhos científicos que tem surgido nas ultimas décadas e abordam a temática de aplicativos para dispositivos móveis, especificamente no ensino da matemática.

Ao considerar a inserção de Tecnologias da Informação e Comunicação no contexto da educação matemática podese promover interação do sujeito com os conteúdos abordados por meio de ferramentas interativas que também simbolizam avanços no processo de ensinoaprendizagem. É preciso repensar a sala de aula dos dias atuais, haja visto a diversidade tecnológica acessível ao professor e já presente no cotidiano do estudante. Entretanto, o que tem se constatado é que a utilização destas novas ferramentas para fins educacionais muitas vezes tem ocorrido de maneira informal, ou seja, fora do ambiente escolar.

A Base Nacional Comum Curricular do
Ensino Médio (BRASIL, 2017) levanta
elementos interessantes do ponto de vista da
cognição que se deseja dos estudantes a
medida que avançam em seus estudos e
destaca que é preciso promover uma
educação integral, que possibilite o
desenvolvimento do estudante de forma
plena para as mais variadas formas de
conhecimento, não limitando a repetição de
processos, mas impulsionando o
desenvolvimento intelectual, emocional,
cultural, físico e social. E o uso de recursos de
tecnologia alia-se a essa proposta, pode-se
tornar as aulas atraentes e interessantes.

É papel do professor avaliar, incorporar e quando possível também desenvolver ferramenta e recursos de tecnologia que promovam melhorias para o ensino. Diante deste quadro, o objetivo deste trabalho é desenvolver e apresentar um aplicativo para dispositivos móveis com recursos de Realidade Aumentada e levantar as percepções de professores de matemática quanto ao uso desta ferramenta. O público alvo é composto de professores de matemática e graduandos de um curso de licenciatura plena em matemática que participaram de uma oficina temática promovida em uma universidade estadual no Paraná. Os nomes dos participantes e também da instituição envolvida não será divulgado com intuito de garantir o anonimato.

\section{APONTAMENTOS SOBRE O TEMA}

Agregar estas novas ferramentas tecnológicas ao ensino de matemática pode ser um desafio com alguns óbices, entretanto é uma ação necessária para os dias atuais, principalmente nas séries finais da Educação Básica, pois os conteúdos e interações ali proporcionados devem preparar o discente para o futuro, garantindo também o domínio das ferramentas tecnológicas. Para Kenski (2007, p.21), "A evolução tecnológica não se restringe apenas aos novos usos de determinados equipamentos e produtos. Ela altera comportamentos". Além do mais, Castro (2018, p. 18) destaca que essas tecnologias tem um poder de encantamento nas mais diferentes idades, viabilizando "[...] aos alunos conversar, pesquisar, bem como interagir com alunos da mesma ou de outras cidades, até mesmo países, conforme seu próprio interesse e ritmo.". Ressaltando o que Meirelles e Tarouco (2005, p.632) indicam como: "um contexto autêntico e apropriado de uso", desenvolto a partir de uma interação personalizada entre o estudante e a tecnologia.

Neste sentido, recursos de tecnologia como a Realidade Aumentada (RA) destacamse e ganham propulsão à medida que vão gerando interesse em empresas de tecnologia. Essa difusão tecnológica só é possível por meio dos avançados equipamentos móveis como o tablet, smartphone, óculos de realidade virtual, entre outros, que também são ferramentas de cunho prodigioso para à educação, graças a facilidade de acesso (KIRNER; SISCOUTTO, 
2007). Corroborando com este pensamento, Denardin e Manzano (2017, p.08) afirmam que: "o uso da RA disponibiliza elementos que facilitam a contextualização dos conteúdos trabalhados em aula, uma vez que os apresenta a partir de múltiplas perspectivas.".

O surgimento dos primeiros aparatos de Realidade Virtual como: luvas interativas, simuladores, capacetes impulsionaram a criação de softwares de interação, mas segundo Kirner e Siscoutto (2007), a Realidade Aumentada surgiu apenas por volta dos anos 90 viabilizada pela ideia de sobrepor por meio tecnológico objetos virtuais em ambientes reais. Todavia apenas nos últimos anos é que este recurso ganhou destaque, diante de dispositivos móveis cada vez mais avançados e de computadores com processadores de alto desempenho.

Em suma, a Realidade Aumentada (RA) ou Augmented-Reality (AR) possibilita a inserção de objetos virtuais num cenário com imagens reais que foram capturadas pelo computador (KIRNER;KIRNER, 2011; DENARDIN; MANZANO, 2017; SCANDOLIERI; SISCOUTTO, 2014). E por adicionar informações a um cenário real surgi o conceito de "aumentada" porque pode gerar no usuário a "sensação" de que aquele ambiente retratado pela computação contém novas informações, aumentando assim a sua percepção. Estimulando o utilizador de forma diferenciada porque, de acordo com Bulla e Rosa, "a RA nos proporciona uma experiência qualitativamente diferente de uma decorrida na tela de um computador, em virtude dos objetos estarem "presos" ou "amarrados" apenas à realidade cibernética"( 2017, p. 300).

Provavelmente as ferramentas tecnológicas que implementam a RA poderão fundir-se ao cotidiano, mas é a partir de interações que se pode constatar e refinar quais as formas mais adequadas de uso desta ferramenta para o ensino, especialmente quando aliada aos dispositivos móveis. Bulla e Rosa (2017, p. 317) fazem um relato e apontam que: "[...] a sensação de que o vínculo da RA com aparelhos eletrônicos de interfaces naturais (especificamente hápticas) (smartphones e/ou tablets, por exemplo) nos possibilitaram um "pensar" diferente".

Desta forma, destaca-se que a criação de aplicativos para dispositivos móveis que fazem uso de recursos adicionais como a Realidade Aumentada ganha força e impulsiona melhorias nas interações durante o aprendizado. Hara e Siscoutto (2018) ao desenvolverem um aplicativo com recursos de RA para o estudo de estrutura de dados indicam que, dentre as muitas contribuições para o aprendizado, estão: a forma interativa e inovadora apresentada através deste tipo de aplicativo; a promoção do conhecimento e a possibilidade de imersão dos alunos no assunto; a difusão deste tipo de tecnologia no meio educacional. No âmbito do ensino médio, Macedo, Silva e Buriol (2016) elencam três aspectos positivos do uso de dispositivos móveis com recursos de RA: a receptividade dos alunos quanto ao uso destas ferramentas de tecnologia como recurso pedagógico; a motivação causada pela RA e a eficiência na visualização de objetos 3D possibilitada por estas ferramentas.

Atualmente é possível desenvolver ferramentas com recursos de RA de forma cada vez mais intuitiva, a medida em que se tem um avanço exploratório desta tecnologia. Scandolieri e Siscoutto (2014) exploram o potencial das aplicações de RA para usuários sem conhecimentos prévios de desenvolvimento de software, apresentando possibilidades de criação de objetos interativos por meio de programação visual, grafo de cena e widgets, que solicita do usuário apenas um computador com acesso a internet, webcam e um marcador impresso.

\section{DESENVOLVIMENTO E APRESENTAÇÃO DE UM APLICATIVO}

O desenvolvimento de um aplicativo que permite a interação com a RA deu-se por meio da plataforma Unity3D (UNITY, 2018), que viabiliza a produção dos mais variados tipos de software em duas ou três dimensões 
como: jogos, banner ou aplicativos de interação. A metodologia empregada para este desenvolvimento é a mesma utilizada em jogos multiplataforma e contempla cinco etapas: Instalação da plataforma Unity, Criação de novo projeto, Inserção de objetos de cena, Funcionalidades e Compilação e Teste do aplicativo.

A interface de usuário do aplicativo apresenta um menu composto por três botões: Ligar Câmera, Créditos do trabalho e Sair. Ao escolher o botão "ligar câmera" o usuário poderá iniciar a interação com os recursos disponíveis no aplicativo. A câmera do smartphone, ou tablet será acionada e o software irá examinar as imagens capturadas. Toda a vez que um cartão de leitura for reconhecido, o software apresenta na tela um dos cinco sólidos regulares. Para tanto, desenvolveu-se cinco cartões de leitura, um para cada sólido de Platão, e a Figura 1 ilustra a situação descrita na qual o cartão de leitura posicionado na frente da câmera do smartphone gerou o sólido específico, no caso, um hexaedro.

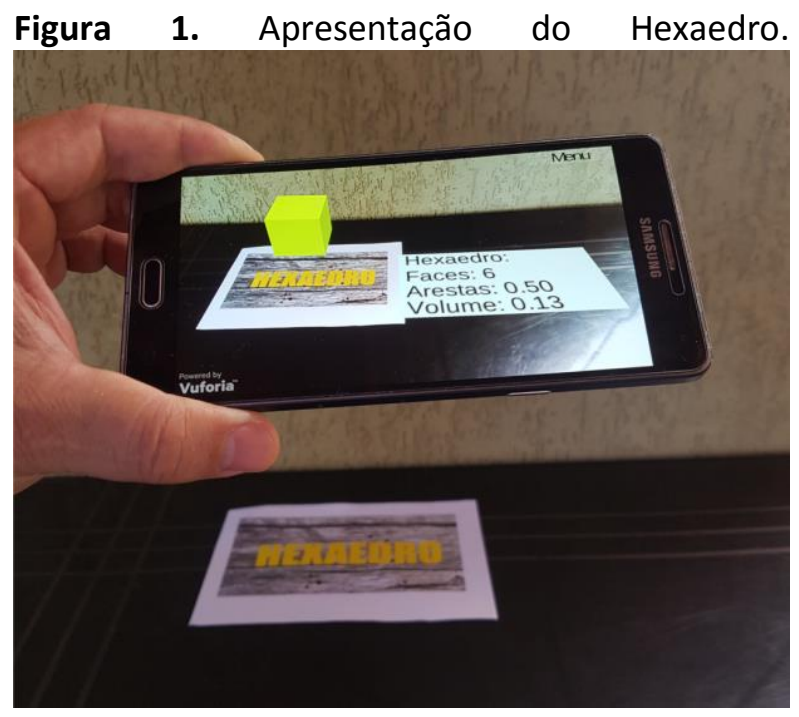

Fonte: Autor.

Além da apresentação do sólido, de acordo com o cartão de leitura, o software também mostra uma etiqueta virtual à direita do sólido virtual que foi desenvolvida para exibir informações específicas.

A interação com os sólidos ocorre por meio de duas funções "click", ilustradas na Figura 2. Aciona-se o primeiro comando "click" toda vez que o usuário toca a região da tela onde o objeto está posicionado. E desta ação por parte do usuário, o software traz uma interface com a planificação do sólido. O segundo comando ocorre quando o usuário toca a região da tela onde a etiqueta virtual traz uma interface com informações sobre volume e área do sólido.

Figura 2. Comando de Click

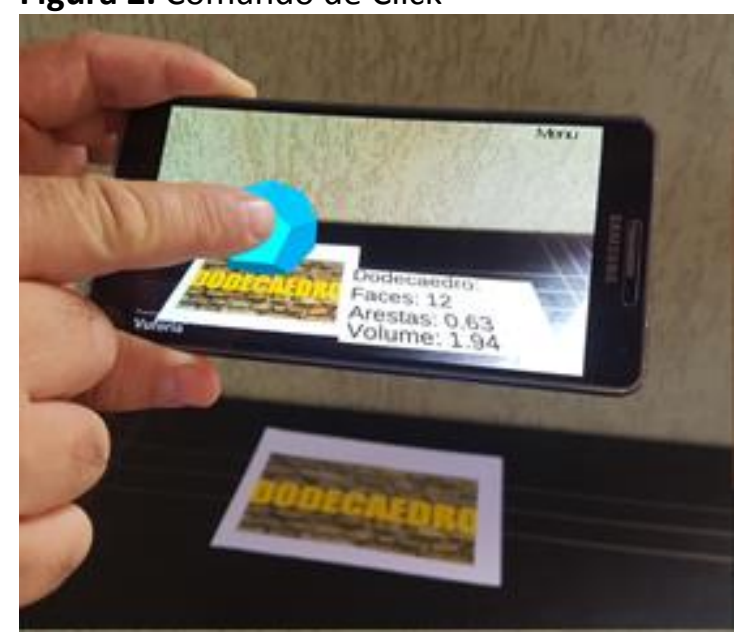

Fonte: Autor.

Além do comando de "click", outro comando implementado e intuitivo é o comando denominado por "pinça na tela". Este comando ocorre quando o usuário posiciona dois dedos sobre a tela, afastandoos ou aproximando-os. Ao acionar este comando, sobre o a região onde o sólido se encontra na tela, o usuário poderá aumentar ou diminuir o tamanho do objeto. Quando se aproxima os dedos ocorre à redução do sólido, como apresentada pela Figura 3. Por sua vez, os dados da etiqueta sofrem alteração, recalculando de modo imediato os valores do volume e da aresta do sólido. 
Figura 3. Comando pinça: redução do tamanho do sólido.

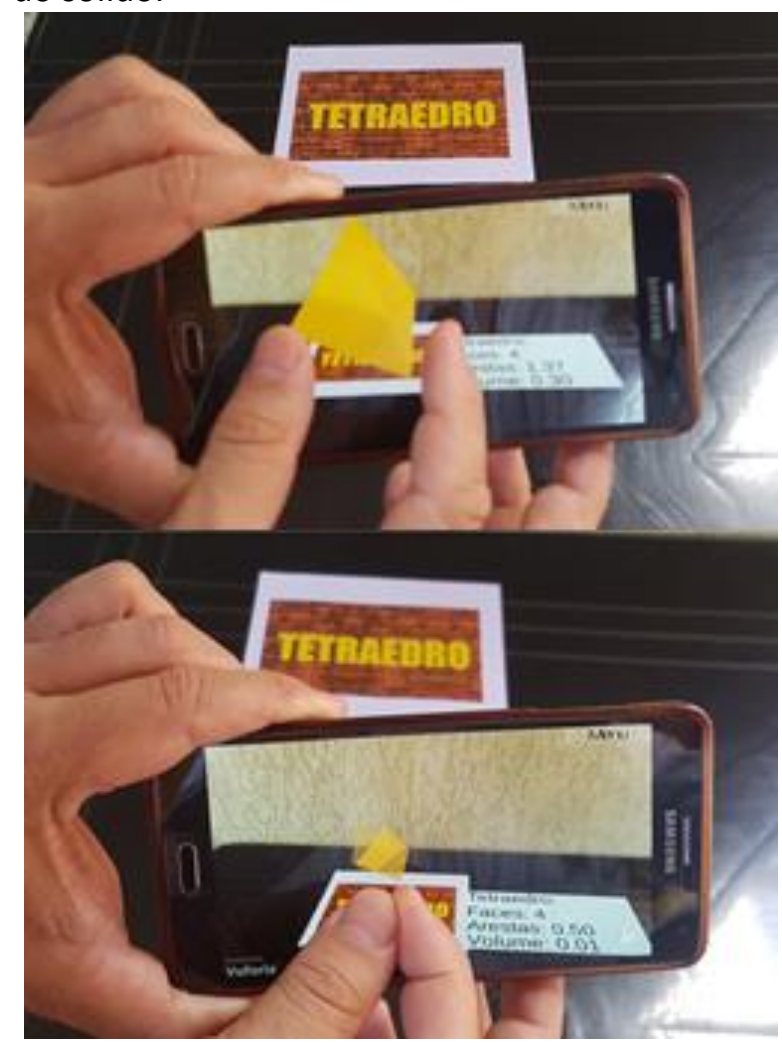

Fonte: Autor.

\section{PERCURSO METODOLÓGICO}

A pesquisa transcorreu durante um encontro educacional da área de educação matemática promovido por uma universidade pública do Estado do Paraná, em que foi promovida uma oficina temática, com um grupo composto de professores de matemática e graduandos de um curso de licenciatura plena em matemática. A oficina foi dividida em três etapas, sendo que a primeira permitiu aos participantes conhecerem aplicativos e os recursos de Realidade Aumentada; permitindo que partir de dispositivos móveis ou de computadores os participantes tivessem acesso à interação e abordagem de conceitos de matemática do ensino básico como: quatro operações, álgebra e geometria. Na segunda etapa todos os participantes puderam interagir com estas ferramentas tecnológicas, e, especialmente, com o aplicativo desenvolvido. Cada um dos participantes pôde manipular e interagir com o software, o tempo estipulado para esta interação do grupo com o software levou cerca de 30 minutos. Na terceira etapa, aplicou-se um questionário semiaberto com intuito de identificar as percepções do público investigado e a relevância do aplicativo desenvolvido. Este questionário compõe-se basicamente de questões que procuram levantar dados pertinentes a postura do professor em relação ao uso de tecnologias em sala de aula.

\section{APRESENTAÇÃO E ANÁLISE DOS RESULTADOS}

A análise dos dados revelou que o público entrevistado é composto de 5 (cinco) professores em exercícios que representam cerca de $40 \%$ e 8 (oito) alunos de licenciatura em matemática que representam $60 \%$ do público entrevistado. Apurou-se que 10 (dez) dos entrevistados, cerca de $77 \%$, opinaram como relevante a inserção de aplicativos como recurso de apoio a abordagem de conteúdos nas aulas de Matemática, em destaque pelo Gráfico 1. Esses resultados estão em paridade com os apresentados por Macedo, Silva e Buriol (2016) que desenvolveram e promoveram a interação com aplicativo móvel com recursos de RA, segundo os autores o público se mostrou motivado e o aplicativo foi bem avaliado pelos estudantes. Vale ressalta que aqui quando se fala em motivação, pretende-se argumentar que ouve interesse na participação das atividades, questionamentos, discussões e envolvimento de forma voluntária por parte dos participantes. 
Gráfico 1. A Relevância dos aplicativos segundo os entrevistados

\section{Relevância sobre o uso de aplicativos em sala de aula}

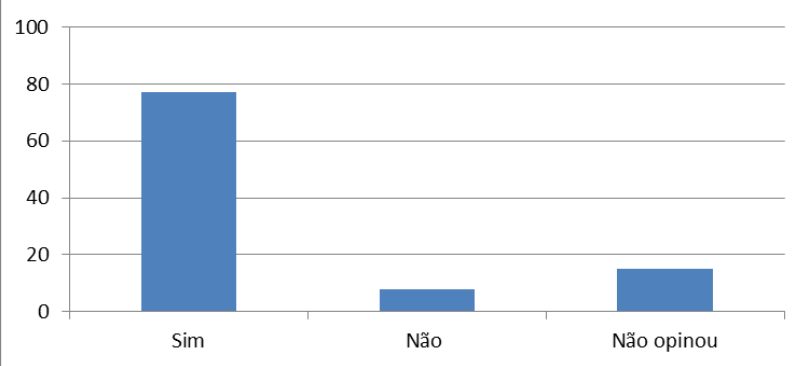

Fonte: Autor.

A respeito das ferramentas tecnológicas que foram apresentadas durante a oficina (aplicativos, jogos virtuais e ferramentas com RA) apenas 4 (quatro) participantes, cerca de $30 \%$, disseram não conhecer ou não ter tido contato com estas ferramentas, os outros, 9 (nove) participantes, representando $70 \%$ indicaram já ter tido contato prévio, seja por meio de alguma interação ou informações de jornais ou revistas. Apresentando também um quadro com resultados próximos aos obtidos por Macedo, Silva e Buriol (2016), quando estes perguntam a estudantes do ensino médio sobre conhecer algum aplicativo de Realidade Aumentada, de acordo com os autores, cerca de $64 \%$, dos entrevistados já ouviram falar ou tiveram algum contato com estes recursos e apenas $36 \%$ disseram não conhecer. Fato importante que evidencia que estes recursos, têm de alguma forma, chegado ao conhecimento de professores e alunos do ensino básico.

Entretanto, ao analisar somente os relatos dos professores que já estão em exercício, por volta $60 \%$ destes descreveram já ter usado pelo menos um aplicativo em dispositivo móvel durante a sua prática docente. Este resultado é conflitante quando comparado ao fato deste que, estes mesmos professores apresentaram no questionário uma auto-reflexão negativa, quanto ao domínio e conhecimento sobre recursos de tecnologia em sua prática docente. Isso dispara um alerta para o fato de que estes professores podem não utilizar estes recursos com eficiência. Refletindo a necessidade de políticas que estimulem à formação continuada, para o desenvolvimento de saberes institucionais que mobilizem o professor a utilizar tecnologias em suas práticas docentes com excelência. Fato importante também a ser mencionado é que nos relatos dos professores os aplicativos mais utilizados em suas práticas são: Geogebra e Youtube. Reportando que estes professores ainda não tiveram contato com outros aplicativos dessa natureza para z abordagem de conteúdos de matemática que apresentem recursos de RA. Indicando que alguns recursos de tecnologia móvel ainda não ganharam destaque na prática pedagógica destes professores ou que estes recursos ainda não foram muito difundidos entre o grupo entrevistado. Entretanto importa destacar que estes dispositivos de tecnologia nas abordagens educacionais possuem grande potencial (HARA; SISCOUTTO, 2018).

Quanto ao uso de aplicativos para dispositivos móveis que possuem recursos de RA, todos os participantes consideraram como relevante o seu uso para as aulas de matemática, de acordo com dados presentes no Gráfico 2, em que 5 (cinco) participantes, algo em torno de 40\%, apontam como relevante a utilização para qualquer conteúdo, os outros 8 (oito) participantes, $60 \%$, apontam ser relevantes, mas ressalvam que apenas para determinados conteúdos, como os sólidos de Platão.

Gráfico 2. Relevância sobre o uso de aplicativos com Realidade Aumentada

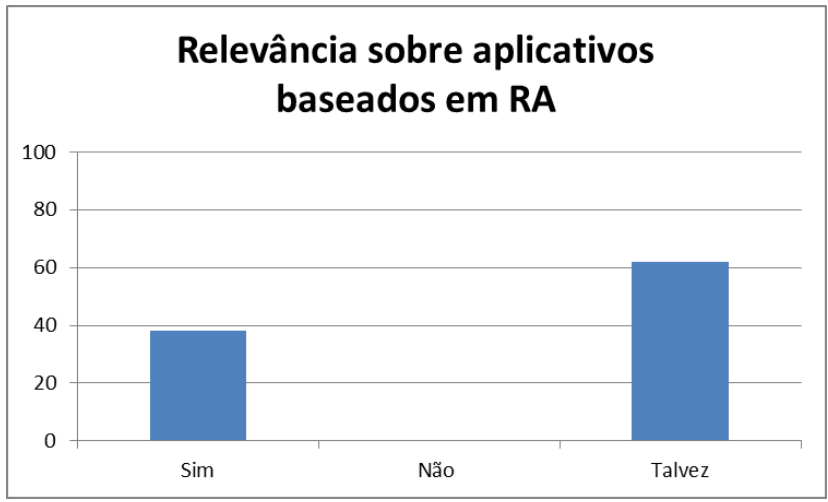

Fonte: Autor. 
Este resultado vai ao encontro dos trabalhos de Kirner e Kirner (2011) e Denardin e Manzano (2017), que indicam uma tendência de aceitação da Realidade Aumentada em processos educacionais. Reforçando a ótica de Denardin e Manzano ao afirmarem que: "a RA não deve ser vista apenas como uma forma de complementar o material didático, mas sim como parte dele que contribui de forma efetiva para a construção de conhecimento por parte dos alunos." (2017, p.09).

A RA pode servir como um apoio educacional importante, mas que não é considerada pelos professores como uma ferramenta "salvadora e revolucionária" de todas as práticas docentes, pensamento que vai de encontro aos aportes teóricos dos pesquisadores Meirelles e Tarouco (2005), Kenski (2007) e Castro (2018). Reforçando esta ideia, Castro (2018, p.206) recomenda que, para empregar estas tecnologias de modo a surtir efeitos satisfatórios, as primeiras ações devem ser no tocante a preparar o professor através de boas formações, tornando-o apto no domínio destas ferramentas, pois de acordo com a autora "não bastam apenas equipamentos e serviço de manutenção, faz-se prioritário um preparo conceitual e técnico de todas as pessoas envolvidas na implementação e utilização dessas ferramentas no ambiente escolar.".

O tema abordado desperta interesse por parte dos professores e isso foi apurado a partir das falas que indicam satisfação em interagir como ferramentas diferenciadas como são os aplicativos para dispositivos móveis e, em especial, o direcionado com recursos de RA. Estes resultados também foram obtidos por (HARA; SISCOUTTO, 2018) ao analisar o aplicativo para dispositivos móveis "SEDRA", que possui recursos de RA, os resultados obtidos também através de um grupo de professores indicam que o aplicativo despertou a motivação e a curiosidade por parte dos participantes da pesquisa, mas que sempre há a possibilidade de melhorias em termos de usabilidade, para que se possa atender melhor o usuário.

\section{CONSIDERAÇÕES FINAIS}

Pode-se constatar que diante das inúmeras tecnologias disponíveis para a prática do professor de matemática na abordagem de conteúdos, a Realidade Aumentada mostra-se uma opção viável para as práticas pedagógicas quando implementada em dispositivos móveis via aplicativos específicos.

Neste estudo, evidencia-se que o professor de matemática demonstra interesse em utilizar ferramentas tecnológicas inovadoras, entretanto muitos relataram estar despreparados para tal. Isso sugere que há urgência de inovação nas políticas de capacitação e renovação da prática docente quanto ao uso de tecnologias móveis. Outro fato importante a ser destacado, é a utilização de aplicativos para dispositivos móveis mostrar-se mais tangível e vantajosa financeiramente que as salas de informática habituais, esta ferramenta é vista pelos professores com um recurso que não deve ser adicionado de forma contínua, como única forma de ensino-aprendizagem.

Destaca-se que pode haver melhorias no aplicativo principalmente quanto a interface do usuário, alguns participantes relataram dificuldade ao explorar o aplicativo, indicando a possibilidade de inserção de uma janela no formato de um guia que reúna os comandos de interação do aplicativo. Entretanto o aplicativo mostrou-se relevante para os envolvidos na pesquisa, despertando o interesse na utilização para a prática pedagógica. Os professores entrevistados deixaram alguns questionamentos de como seria trabalhar com outros conteúdos da matemática como: aritmética, álgebra e, até mesmo, o cálculo diferencial e integral. $\mathrm{O}$ que se pode apontar como assuntos proeminentes e possíveis impulsionadores de novas pesquisas em torno desta temática. 


\section{REFERÊNCIAS}

BRASIL. Ministério da Educação. Base Nacional Comum Curricular. Terceira Versão. Brasília: MEC, 2017.

BULLA, F. D.; ROSA, M. O Design de tarefasmatemáticas-com-realidade-aumentada:

uma autorreflexão sobre o processo. Acta Scientiae, v. 19, n. 2, 2017. Disponível em: http://www.periodicos.ulbra.br/index.php/ac ta/article/view/3036/2304. Acesso em: 20 out. 2018.

CASTRO, A. L. Matemática e o currículo da era digital: os desafios para a inovação na prática educativa. 2018. Tese (Doutorado) UNESP, Bauru, 2018.

DENARDIN, L.; MANZANO, R. C.. Desenvolvimento, utilização e avaliação da realidade aumentada em aulas de física. RENOTE, v. 15, n. 2, 2017. https://doi.org/10.22456/1679-1916.79258

HARA, M. M. S.; SISCOUTTO, R. A.. Objetos de aprendizagem para ensino de estruturas de dados fazendo uso de realidade aumentada: sedra. Colloquium Exactarum, v. 10, n. 1, 2018.

https://doi.org/10.5747/ce.2018.v10.n1.e224

KENSKI, V. M. Educação e tecnologias: o novo ritmo da educação. 7. ed. São Paulo: Papirus, 2007.

KIRNER, C.; KIRNER, T. G. Evolução e tendências da realidade virtual e da realidade aumentada. In: RIBEIRO, M. W. S.; ZORZAL, E. R. (org.) Realidade virtual e aumentada: Aplicações e Tendências. Uberlandia: SBC, , 2011. Cap, v. 1, p. 10-25

KIRNER, C.; SISCOUTTO, R. A. Fundamentos de Realidade Virtual e Aumentada. In: Realidade virtual e aumentada: conceitos, projeto e aplicações. Livro do IX Symposium on Virtual and Augmented Reality, Petrópolis (RJ), Porto Alegre: SBC. 2007. p. 2-29.
MACEDO, A. C.; SILVA, J. A.; BURIOL, T. M. Usando Smartphone e Realidade aumentada para estudar Geometria espacial. RENOTE. Revista Novas Tecnologias na Educação, v. 14 n. 2, p. 1-10, 2016. https://doi.org/10.22456/1679-1916.70688

MEIRELLES, L. F. T.; TAROUCO, L. MR. Framework para aprendizagem com mobilidade. In: BRAZILIAN SYMPOSIUM ON COMPUTERS IN EDUCATION (SIMPÓSIO BRASILEIRO DE INFORMÁTICA NA EDUCAÇÃO-SBIE. 16., . 2005, Juis de Fora. Anais [...]. Juis de Fora: UFJF, 2005. p. 623633. Disponível em: http://www.brie.org/pub/index.php/sbie/article/view/446/ 432. Acesso em: 10 out. 2018.

SCANDOLIERI, B. R.; SISCOUTTO, R. A.. Desenvolvimento de Aplicações de Realidade Aumentada Utilizando Programação Visual. Colloquium Exactarum, v.6, n. 2, 2014. https://doi.org/10.5747/ce.2014.v06.n2.e075

UNITY. Unity, 2018. Site da empresa Unity. Disponível em: https://unity3d.com/pt. Acesso em: 10 ago. 2018. 